\title{
ON A PROBLEM OF NIEDERREITER AND ROBINSON ABOUT FINITE FIELDS
}

\author{
DAQING WAN
}

(Received 26 October 1984; revised 2 April 1985)

\begin{abstract}
In this article, we prove that for a finite field $F_{q}$ with even $q>3$, any complete mapping polynmial of $F_{q}$ has reduced degree at most $q-3$. This is a solution to a problem of Niederreiter and Robinson about finite fields.;
\end{abstract}

1980 Mathematics subject classification (Amer. Math. Soc.): 12 C 05.

In this article, we consider a special class of mappings of a finite field into itself. We start with the following definition.

DEFINITION. Let $f(x)$ be a polynomial over a finite field $F_{q}$.

(1) If the mapping $c \in F_{q} \rightarrow f(c)$ is a bijection, then $f(x)$ is called a permutation polynomial of $F_{q}$.

(2) If both $f(x)$ and $f(x)+x$ are permutation polynomials of $F_{q}$, then $f(x)$ is called a complete mapping polynomial of $F_{q}$.

(3) The degree of the reduction of $f(x)$ modulo $\left(x^{q}-x\right)$ is called the reduced degree of $f(x)$. It is unique and is always less than $q$.

Dickson [1] proved that a permutation polynomial $f(x)$ of $F_{q}$ has reduced degree at most $q-2$.

Recently, Niederreiter and Robinson [2] proved that for a finite field $F_{q}$ with odd $q>3$, any complete mapping polynomial $f(x)$ has reduced degree at most $q-3$. They indicated that it would be of interest to determine whether this result holds also for even $q$.

We obtain an affirmative answer to this problem in the following theorem.

(c) 1986 Australian Mathematical Society $0263-6115 / 86 \$ A 2.00+0.00$ 
THEOREM. Let $q=2^{k}>3$. Then any complete mapping polynomial of $F_{q}$ has reduced degree at most $q-3$.

This bound is also in a sense best possible since $f(x)=a x(a \neq 0,1)$ is a complete mapping polynomial of $F_{4}$ of reduced degree 1 .

Proof (of the theorem). It is well known that $F_{q}$ can be identified with the residue class ring $E / 2 E$ for a suitable ring $E$ of algebraic integers in an algebraic number field. Let $\eta$ be the canonical ring homomorphism from $E$ onto $F_{q}=$ $E / 2 E$. Then $\eta$ can be extended to a homomorphism $\eta^{\prime}$ of $E[x]$ to $F_{q}[x]$ $\left(\eta^{\prime}(x)=x\right)$; we still write the map $\eta^{\prime}$ as $\eta$.

Now, let $g$ be a generator of $F_{q}, g_{1}$ be an inverse image of $g$. Then we have

$$
g_{1}^{q-1} \equiv 1(\bmod 2), \quad g_{1}^{i} \not \equiv 1(\bmod 2), \quad \text { for } 0<i<q-1 .
$$

If $g_{1}^{q-1} \not \equiv 1(\bmod 4)$, then

$$
\begin{aligned}
&\left(g_{1}\left(1+2\left(\frac{g_{1}^{q-1}-1}{2}\right)\right)\right)^{q-1} \equiv g_{1}^{q-1}+2 g_{1}^{q-1}(q-1)\left(\frac{g_{1}^{q-1}-1}{2}\right) \\
& \equiv 1+2\left(\frac{g_{1}^{q-1}-1}{2}\right)-2 g_{1}^{q-1}\left(\frac{g_{1}^{q-1}-1}{2}\right) \equiv 1(\bmod 4)
\end{aligned}
$$

and

$$
\eta\left(g_{1}\left(1+2\left(\frac{g_{1}^{q-1}-1}{2}\right)\right)\right)=\eta\left(g_{1}\right)=g .
$$

Hence, without loss of generality, we may suppose that

$$
g_{1}^{q-1} \equiv 1(\bmod 4) \text {. }
$$

For a permutation polynomial $f(x)$ of $F_{q}$, let $F(x)$ be an inverse image of $f(x)$, that is, $\eta(F(x))=f(x)$, and let $S=\left\{g_{1}^{i} \mid 1 \leqslant i \leqslant q-1\right\} \cup\{0\}$.

From the definition, we have

$$
\{\eta(x) \mid x \in S\}=F_{q}, \quad\{\eta(F(x)) \mid x \in S\}=F_{q} .
$$

Hence

$$
\sum_{x \in S} F^{2}(x)=\sum_{x \in S}(x+2 \cdot G(x))^{2},
$$

where $G(x) \in E$ for any $x \in S$. By (2), we have

$$
\sum_{x \in S} F^{2}(x) \equiv \sum_{x \in S} x^{2}(\bmod 4)=g_{1}^{2}\left(\frac{g^{2(q-1)}-1}{g_{1}^{2}-1}\right) \equiv 0(\bmod 4)
$$


by (1). If $f(x)$ is a complete mapping polynomial of $F_{q}$, then both $f(x)$ and $f(x)+x$ are permutation polynomials of $F_{q}$. In terms of (3), this given

$$
\sum_{x \in S} F^{2}(x) \equiv 0(\bmod 4)
$$

and

$$
\sum_{x \in S}(F(x)+x)^{2} \equiv 0(\bmod 4) .
$$

By Dickson's theorem, the permutation polynomial $f(x)$ can be taken in the form

$$
f(x)=a_{q-2} x^{q-2}+a_{q-3} x^{q-3}+\cdots+a_{0}, \quad a_{i} \in F_{1} .
$$

Let $F(x)$ be an inverse image of $f(x)$ such that

$$
F(x)=b_{q-2} x^{q-2}+b_{q-3} x^{q-3}+\cdots+b_{0}, \quad b_{i} \in E .
$$

From (4) and (5), we have

$$
\begin{aligned}
0 & \equiv \sum_{x \in S}(F(x)+x)^{2}=\sum_{x \in S} F^{2}(x)+\sum_{x \in S} x^{2}+2 \sum_{x \in S} x F(x) \\
& \equiv 0+0+2 \sum_{x \in S} b_{q-2} x^{q-1} \equiv-2 b_{q-2}(\bmod 4) .
\end{aligned}
$$

Hence, $b_{q-2} \equiv 0(\bmod 2)$, and $a_{q-2}=\eta\left(b_{q-2}\right)=0$, that is, $f(x)$ has reduced degree at most $q-3$, and the theorem is proved.

The author is grateful to Professor Ko Chao and Professor Sun Oi for their kind guidance.

\section{References}

[1] L. E. Dickson, Linear groups (Dover, New York, 1958).

[2] H. Niederreiter and K. H. Robinson, 'Complete mappings of finite fields,' J. Austral. Math. Soc. (Ser. A) 33 (1982), 197-212.

\section{Department of Mathematics}

Sichuan University

Chengdu

China 